\title{
Ancylostoma ceylanicum Hookworm in Myanmar Refugees, Thailand, 2012-2015
}

\author{
Elise M. O'Connell, Tarissa Mitchell, Marina Papaiakovou, Nils Pilotte, Deborah Lee, \\ Michelle Weinberg, Potsawin Sakulrak, Dilok Tongsukh, Georgiette Oduro-Boateng, \\ Sarah Harrison, Steven A. Williams, William M. Stauffer, ${ }^{1}$ Thomas B. Nutman ${ }^{1}$
}

\begin{abstract}
Medscape ACTIVITY

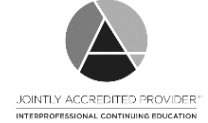
American Nurses Credentialing Center (ANCC), to provide continuing education for the healthcare team.

Medscape, LLC designates this Journal-based CME activity for a maximum of 1.00 AMA PRA Category 1 Credit(s) ${ }^{\mathrm{TM}}$. Physicians should claim only the credit commensurate with the extent of their participation in the activity.

All other clinicians completing this activity will be issued a certificate of participation. To participate in this journal CME activity: (1) review the learning objectives and author disclosures; (2) study the education content; (3) take the post-test with a $75 \%$ minimum passing score and complete the evaluation at http://www.medscape.org/journal/eid; and (4) view/print certificate. For CME questions, see page XXXX.
\end{abstract}

Release date: July 11, 2018; Expiration date: July 11, 2019

Learning Objectives

Upon completion of this activity, participants will be able to:

- Evaluate the epidemiology of hookworm infection among Myanmar refugees living in 3 camps along the Thailand-Myanmar border, based on a cohort study

- Analyze the response to treatment of hookworm infection assessed through molecular analyses among Myanmar refugees living in 3 camps along the Thailand-Myanmar border, based on a cohort study

- Identify risk factors for hookworm infection among Myanmar refugees living in 3 camps along the Thailand-Myanmar border, based on a cohort stud.

CME Editor

Kristina B. Clark, PhD, Copyeditor, Emerging Infectious Diseases. Disclosure: Kristina B. Clark, PhD, has disclosed no relevant financial relationships.

CME Author

Laurie Barclay, MD, freelance writer and reviewer, Medscape, LLC. Disclosure: Laurie Barclay, MD, has disclosed the following relevant financial relationships: owns stock, stock options, or bonds from Pfizer.

Authors

Disclosures: Elise M. O'Connell, MD; Tarissa Mitchell, MD; Marina Papaiakovou, MSc; Nils Pilotte, MS; Deborah Lee, MPH; Michelle Weinberg, MD, MPH; Potsawin Sakulrak, BS; Dilok Tongsukh, MS; Georgiette Oduro-Boateng, BS; Sarah Harrison, BS; Steven A. Williams, PhD; William M. Stauffer, MD; and Thomas B. Nutman, MD, have disclosed no relevant financial relationships. This article may include discussion of off-label uses of drugs, mechanical devices, biologics, or diagnostics approved by the FDA for use in the United States and investigational drugs, mechanical devices, biologics, or diagnostics not approved by the FDA for use in the United States.

Author affiliations: National Institutes of Health, Bethesda, Maryland, USA (E.M. O'Connell, G. Oduro-Boateng, S. Harrison, T.B. Nutman); Centers for Disease Control and Prevention, Atlanta, Georgia, USA (T. Mitchell, D. Lee, M. Weinberg, W.M. Stauffer); Smith College, Northampton, Massachusetts, USA (M. Papaiakovou, N. Pilotte, S.A. Williams); University of Massachusetts, Amherst, Masschusetts, USA (N. Pilotte,
S.A. Williams); International Organization for Migration, Mae Sot, Thailand (P. Sakulrak, D. Tongsukh); University of Minnesota Medical School, Minneapolis, Minnesota, USA (W.M. Stauffer)

DOI: https://doi.org/10.3201/eid2408.180280

${ }^{1}$ These authors contributed equally to this article. 
During 2012-2015, US-bound refugees living in MyanmarThailand border camps $(n=1,839)$ were surveyed for hookworm infection and treatment response by using quantitative PCR. Samples were collected at 3 time points: after each of 2 treatments with albendazole and after resettlement in the United States. Baseline prevalence of Necator americanus hookworm was $25.4 \%$, Ancylostoma duodenale $0 \%$, and Ancylostoma ceylanicum (a zoonosis) 5.4\%. Compared with $N$. americanus prevalence, A. ceylanicum hookworm prevalence peaked in younger age groups, and blood eosinophil concentrations during $A$. ceylanicum infection were higher than those for $N$. americanus infection. Female sex was associated with a lower risk for either hookworm infection. Cure rates after 1 dose of albendazole were greater for $A$. ceylanicum $(93.3 \%)$ than $N$. americanus $(65.9 \%)$ hookworm $(p<0.001)$. Lower N. americanus hookworm cure rates were unrelated to $\beta$-tubulin single-nucleotide polymorphisms at codons 200 or 167. A. ceylanicum hookworm infection might be more common in humans than previously recognized.

$\mathrm{H}$ ookworm infection affects $>470$ million persons worldwide (1). Childhood infection has been associated with growth stunting, severe anemia, and iron deficiency $(2,3)$. Ancylostoma duodenale and Necator americanus hookworms are believed to be the most prevalent species that infect humans. Infections with these species are acquired by transdermal penetration of the hookworm larvae or by the fecal-oral route (A. duodenale hookworm only), and infection is limited to humans (4). Dogs and cats infected with Ancylostoma ceylanicum hookworm have been found in close association with human populations (5-9). With use of molecular techniques, an increased number of human A. ceylanicum hookworm infections have been documented in parts of Asia and the Solomon Islands (10-13).

Hookworms and other soil-transmitted helminths are neglected tropical pathogens targeted for worldwide control by the World Health Organization by 2030 (14). This goal is being pursued through the mass administration of benzimidazole compounds (i.e., albendazole, mebendazole). However, the ubiquitous use of a single class of drug in both human and veterinary medicine has raised concern for the emergence of drug resistance (15-20). We report hookworm infection and cure rates in a large cohort of US-bound refugees from Myanmar residing in camps in Thailand along the Myanmar-Thailand border and assess the presence of $\beta$-tubulin mutations that could confer drug resistance among persons with persistent infection.

\section{Materials and Methods}

\section{Recruitment of Participants and Sample Collection}

This investigation was part of a larger program involving refugees living in camps along the Myanmar-Thailand border that was conducted by the Centers for Disease Control and Prevention during 2012-2015 (21). In our analysis, we included $1,839(92 \%)$ of the 2,004 refugees $\geq 6$ months of age from this cohort who provided fecal samples. (Figure 1; online Technical Appendix, https://wwwnc.cdc.gov/EID/ article/24/8/18-0280-Techapp1.pdf). Fecal samples were collected at time point 1 (T1, during the required medical examination for US resettlement), T2 (before departing the refugee camp), and T3 (after US resettlement). All refugees were offered albendazole after fecal specimens were collected to treat presumptive infection with helminths.

\section{Molecular Detection of Hookworm Species}

We performed stool extraction (online Technical Appendix) as previously described (22). For the initial 233 samples, we performed quantitative PCR (qPCR) with primerprobe sets Ad1 (for amplifying A. duodenale) and $\mathrm{Na}$ (for amplifying $N$. americanus) (Table 1) $(23,24)$. Then, we switched to a more sensitive primer-probe set targeting a repetitive element in A. duodenale (Ad2) (24). When qPCR with the Ad2 primer-probe set failed to amplify the samples positive by qPCR with Ad1, we subjected 7 discordant samples to PCR restriction fragment length polymorphism, as previously described (26). We also sequenced these PCR products using standard (Sanger) sequencing technology.
Figure 1. Study design showing collection of fecal samples from and treatment of US-bound Myanmar refugees for hookworm infection, Thailand, 2012-2015. Myanmar refugees $(n=2,004)$ from 3 camps in Thailand (Mae La [camp 1], Mae Ra Ma Luang [camp 2], Mae La Oon [camp 3]) along the MyanmarThailand border were recruited to donate fecal samples and receive treatment (ivermectin and albendazole) for parasitic infections. T1 was the time of the resettlement medical exam, T2 occurred before camp departure, and T3 was a time after resettlement in the United States. Albendazole and ivermectin were given immediately after fecal collection at T1 and T2. T1, time point 1; T2, time point 2; T3, time point 3.
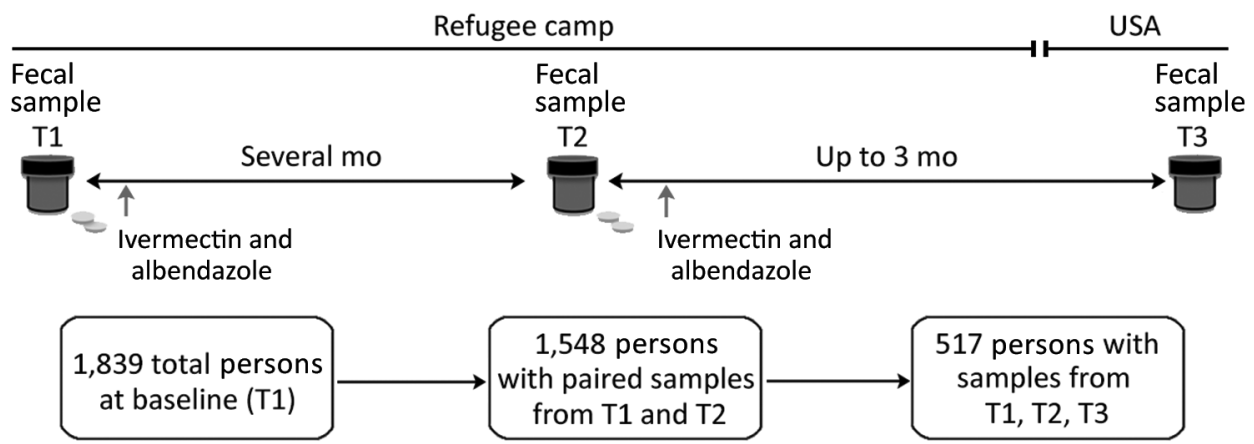

517 persons with mples from

$\mathrm{T} 1, \mathrm{~T} 2, \mathrm{~T} 3$ 
Table 1. Primer-probe sets used to determine genotypes of hookworms present in US-bound Myanmar refugees in camps along Myanmar-Thailand border, Thailand, 2012-2015*

\begin{tabular}{|c|c|c|c|}
\hline $\begin{array}{l}\text { Genome target/primer-probe set name, } \\
\text { sequence type }\end{array}$ & Sequence & $\begin{array}{c}\text { Final concentration, } \\
\mathrm{nmol} / \mathrm{L}\end{array}$ & Reference \\
\hline ITS2/Ad1 & & & (23) \\
\hline Forward primer & 5'-GAATGACAGCAAACTCGTTGTTG-3' & 900 & \\
\hline Reverse primer & 5'-ATACTAGCCACTGCCGAAACGT-3' & 900 & \\
\hline Probe & 5'-ATCGTTTACCGACTTTAG-3' & 250 & \\
\hline Repetitive element/Ad2 & & & (24) \\
\hline Forward primer & 5'-GTATTTCACTCATATGATCGAGTGTTC-3' & 900 & \\
\hline Reverse primer & 5'-GTTTGAATTTGAGGTATTTCGACCA-3' & 900 & \\
\hline Probe & 5'-TGACAGTGTGTCATACTGTGGAAA-3' & 250 & \\
\hline Repetitive element/Ac & & & (25) \\
\hline Forward primer & 5'-CAAATATTACTGTGCGCATTTAGC-3' & 900 & \\
\hline Reverse primer & 5'-GCGAATATTTAGTGGGTTTACTGG-3' & 900 & \\
\hline Probe & 5'-CGGTGAAAGCTTTGCGTTATTGCGA-3' & 250 & \\
\hline Repetitive element/Na & & & $(24)$ \\
\hline Forward primer & 5'-CCAGAATCGCCACAAATTGTAT-3' & 900 & \\
\hline Reverse primer & 5'-GGGTTTGAGGCTTATCATAAAGAA-3' & 900 & \\
\hline Probe & 5'-CCCGATTTGAGCTGAATTGTCAAA-3' & 250 & \\
\hline SNP200 & & & This \\
\hline Forward primer & 5'-AATGCTACACTCTCTGTTCACCAGTT-3' & 900 & paper \\
\hline Reverse primer & 5'-CGGAAGCAGATATCATACAAAGCTT-3' & 900 & \\
\hline Wild type FAM probe/mutant VIC probe† & 5'-AATACAGATGAGACCT(T/A)CT-3' & $166 ; 231$ & \\
\hline SNP167 & & & This \\
\hline Forward primer & 5'-TCGGGAAGAATACCCTGATAGAAT-3' & 900 & paper \\
\hline Reverse primer & 5'-CTTTTGCTCTTATTTCCATCAATAGGA-3' & 900 & \\
\hline Wild type FAM probe/mutant VIC probe† & 5'-TGTCCTCGT(T/A)TTCC-3' & $125 ; 350$ & \\
\hline
\end{tabular}

\section{Definitions}

Participants whose fecal samples were positive for hookworm DNA by qPCR and became negative at the immediate next time point were considered cured. Those whose fecal samples were negative for hookworm DNA by qPCR but then positive the immediate next time point were considered to have a newly acquired infection. We defined persistent infection as having detectable hookworm DNA at 2 successive time points.

\section{Single-Nucleotide Polymorphism Detection in $\mathbf{N}$. americanus-Positive Samples}

For the participants who were positive for $N$. americanus hookworm at all 3 time points, we tested fecal samples from $\mathrm{T} 1$ and $\mathrm{T} 3$ for $\beta$-tubulin single-nucleotide polymorphisms (SNPs) at codon 200. We also tested refugee
T3 fecal samples for SNP167 by using an allele-specific real-time PCR approach with common primers and SNPspecific probes (Table 1) and defined heterozygosity and homozygosity of SNPs on the basis of change in the cycle threshold, similar to previously described methods (27-29) (online Technical Appendix).

\section{Statistical Analyses}

Unless stated otherwise, we used the geometric mean to measure central tendency. We determined the odds ratios (ORs) of risk factors for infection with $A$. ceylanicum and $N$. americanus hookworms by using a generalized linear model that used overdispersion with binomial distribution and logit link. We performed a maximum likelihood analysis using JMP 12.0.1 (https://www.jmp.com/en_us/home.html). We used these models to test the following parameters: sex,

Table 2. Baseline characteristics of 1,839 US-bound Myanmar refugees in camps along Myanmar-Thailand border, by camp, Thailand, 2012-2015

\begin{tabular}{lccc}
\hline Characteristic & Mae La, camp 1 & $\begin{array}{c}\text { Mae La Oon, } \\
\text { camp 2 }\end{array}$ & $\begin{array}{c}\text { Mae Ra Ma Luang, } \\
\text { camp 3 }\end{array}$ \\
\hline Total participants, no. & 549 & 667 & 623 \\
\hline Sex, no. (\%) & & & \\
$\quad$ F & $261(47.5)$ & $327(49.0)$ & $304(48.8)$ \\
$\quad$ M & $288(52.5)$ & $340(51.0)$ & $319(51.2)$ \\
\hline Age, y, mean (range) & $20.8(0.6-79.9)$ & $19.3(0.86-88.9)$ & $18.9(0.55-83.1)$ \\
Any hookworm infection, no. (\%) & $180(32.8)$ & $173(25.9)$ & $182(29.2)$ \\
Hemoglobin, g/L, geometric mean (range) & $130(71-179)$ & $127(71-180)$ & $126(71-183)$ \\
Eosinophil concentration, $\times 10^{8}$ cells/L, geometric mean (range) ${ }^{*}$ & $3.56(0.01-55.3)$ & $4.27(0.01-107)$ & $5.21(0.01-178)$ \\
\hline
\end{tabular}

${ }^{*}$ Eosinophil concentration reference range: $0-4.5 \times 10^{8}$ cells/L. 


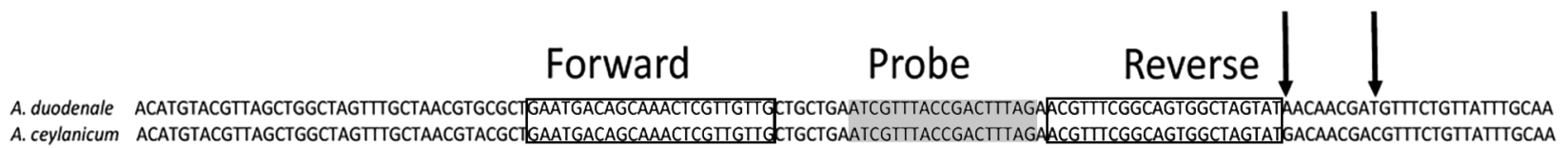

Figure 2. Partial internal transcribed spacer 2 (ITS2) sequences of Ancylostoma duodenale and A. ceylanicum hookworms. Boxes indicate the location of forward and reverse primer binding; gray shading indicates the location of probe binding. These regions of the A. duodenale and A. ceylanicum ITS2 are identical. The locations where the ITS2 sequences differ (arrows) fall outside of the primer and probe binding regions.

camp, age (infants and toddlers $<2$ years of age, children $2-18$ years of age, and adults $>18$ years of age), and coinfections (online Technical Appendix). The cutoff of $<2$ years of age for infants and toddlers was chosen because, after this age, the rates of mouthing, a prominent cause of fecal-oral contamination, decrease $(30,31)$. We performed cure rate comparisons using a 2-tailed Fisher exact test. We compared the geometric mean eosinophil and hemoglobin concentrations of those monoinfected with A. ceylanicum or $N$. americanus hookworm (excluding co-infections involving both hookworms and Strongyloides stercoralis, Ascaris lumbricoides, and Trichuris trichiura roundworms) by the Mann-Whitney test using Prism GraphPad 6.0e (https:// www.graphpad.com/scientific-software/prism/).

\section{Results}

A total of 4,330 fecal samples from 1,839 refugees underwent DNA extraction and multiparallel qPCR. After excluding 4 participants positive for hookworm at $\mathrm{T} 1$ who did not receive albendazole, the number of participants with T1-T2 paired samples totaled 1,548; we had samples from all 3 time points for 517 participants. The geometric mean time between $\mathrm{T} 1$ and $\mathrm{T} 2$ sample collections was 188 (range 48-1,013) days and between T2 and T3 was 46.2 (range 14-413) days. Baseline hookworm infection (any type) in this population was high, ranging from $25.9 \%-32.8 \%$ depending on the camp of residence (Table 2).

\section{Detection of $A$. ceylanicum}

When a highly sensitive primer-probe set specific to the $A$. duodenale genome ( $\mathrm{Ad} 2$ ) was used on samples positive for Ancylostoma DNA by qPCR with primer-probe set Ad1, none was positive. Because of concern that Ad1 might enable the cross-amplification of other Ancylostoma spp., we performed seminested PCR and MvaI and Psp 1406I digestion with 7 samples positive by Ad1 but negative by $\mathrm{Ad} 2$.
Results from restriction fragment length polymorphism PCR indicated the presence of $A$. ceylanicum hookworm (online Technical Appendix Figure); these results were further confirmed by sequencing $(98 \%$ identity to $A$. ceylanicum ribosomal sequence [GenBank accession no. LC036567]). The internal transcribed spacer 2 regions of the $A$. duodenale and $A$. ceylanicum genomes, which the Ad1 primer-probe set aligned with, are identical (Figure 2). Cross-species identification with this primer-probe set has been previously predicted (23), although not previously demonstrated in the literature.

We then determined the prevalence of $A$. ceylanicum hookworm among the refugee population using a primerprobe set specific to a repetitive DNA element in the $A$. ceylanicum genome (Ac; Table 1). When using Ac, the total number of $A$. ceylanicum-positive samples increased from 106 (using the Ad1 set) to 124 (using the Ac set). We tested or retested these samples $(n=124)$ by qPCR using the Ad2 primer-probe set, and $0 \%$ were positive. All samples positive by qPCR with Ad1 were positive by qPCR with Ac.

\section{Response to Treatment}

Baseline prevalence of $N$. americanus (26.3\%) hookworm was higher than that of A. ceylanicum (5.3\%) hookworm among all participants $(\mathrm{n}=1,839)$; likewise, prevalence of $N$. americanus $(25.4 \%)$ species was higher than that of A. ceylanicum (5.4\%) species among all participants who gave paired T1-T2 fecal samples $(\mathrm{n}=1,548)$ (Table 3$)$. Refugees in their sixth (50-59 years) and seventh (60-69 years) decades of life had the highest $N$. americanus hookworm prevalence ( $>40 \%$ positive), and refugees in their third decade of life had the highest $A$. ceylanicum hookworm prevalence (9\%) (Figure 3).

A. ceylanicum infection had a higher cure rate than did $N$. americanus infection (Table 3 ); $92.8 \%$ of $A$. ceylanicum hookworm-infected refugees were cured by $\mathrm{T} 2$, despite the

Table 3. Hookworm prevalence and cure rate in US-bound Myanmar refugees in camps along Myanmar-Thailand border with paired samples, by time point, Thailand, 2012-2015*

\begin{tabular}{|c|c|c|c|c|c|}
\hline \multirow[b]{2}{*}{ Species } & \multirow{2}{*}{$\begin{array}{c}\mathrm{T} 1, \mathrm{n}=1,548 \\
\text { Baseline prevalence, } \\
\text { no. }(\%)\end{array}$} & \multicolumn{2}{|c|}{$T 2, n=1,548$} & \multicolumn{2}{|c|}{$\mathrm{T} 3, \mathrm{n}=517$} \\
\hline & & Cure, no. (\%) & $\begin{array}{c}\text { Persistent infection, } \\
\text { no. }(\%)\end{array}$ & Cure, no. (\%) & $\begin{array}{c}\text { Persistent infection, } \\
\text { no. }(\%)\end{array}$ \\
\hline Ancylostoma ceylanicum & $83 / 1,548(5.4)$ & $77 / 83(92.8)$ & $6 / 83(7.2)$ & $7 / 7(100)$ & 0 \\
\hline Necator americanus & $393 / 1,548(25.4)$ & $271 / 393(69)$ & $122 / 393(31)$ & $21 / 50(42)$ & $29 / 50(58)$ \\
\hline
\end{tabular}



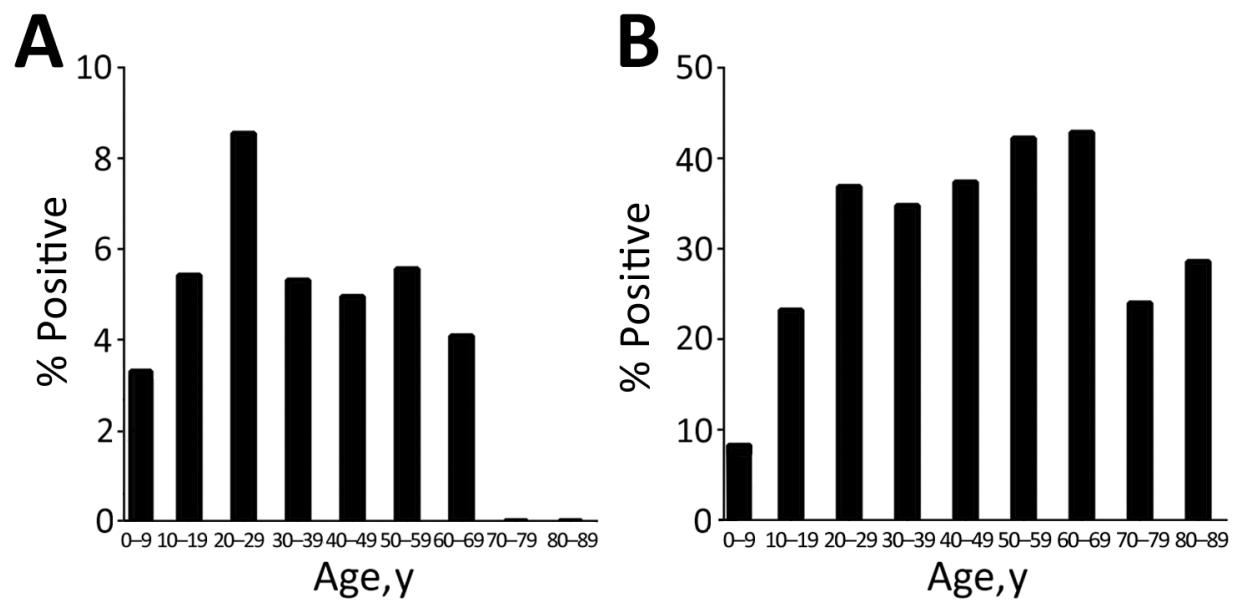

Figure 3. Baseline prevalence of hookworm infections in 1,839 US-bound Myanmar refugees at 3 camps along the Myanmar-Thailand border, by age group, Thailand, 2012-2015. A) Ancylostoma ceylanicum hookworm. B) Necator americanus hookworm.

relatively long time that elapsed between $\mathrm{T} 1$ and $\mathrm{T} 2$. Of the samples paired for $\mathrm{T} 2$ and $\mathrm{T} 3$, all 7 A. ceylanicum hookworm-infected refugees at T2 were cured by T3. At T2, the $N$. americanus infection cure rate was $69 \%$, and after the second administration of albendazole at T3, 42\% (21/50) were cured, resulting in 29 participants with persistent $N$. americanus infection at resettlement in the United States. Combining cure rates across all time points, the overall $A$. ceylanicum hookworm cure rate was $93.3 \%(84 / 90)$, higher than that for $N$. americanus hookworm $(65.9 \%, 292 / 443 ; \mathrm{p}<0.001)$.
In total, 151 refugees had persistent $N$. americanus infections, and 6 refugees had persistent $A$. ceylanicum infections. At T2, the hookworm genomic DNA relative quantity in fecal samples of those with persistent $N$. americanus infections $(\mathrm{n}=122)$ decreased significantly $(\mathrm{p}<0.001)$ (Figure 4). Of the 6 persons with persistent $A$. ceylanicum infections at T2, $4(67 \%)$ had a decrease and $2(33 \%)$ an increase in hookworm genomic DNA in their fecal samples. Those infected with $N$. americanus hookworm at T2 and T3 $(n=29)$ had no significant decrease in parasite genomic
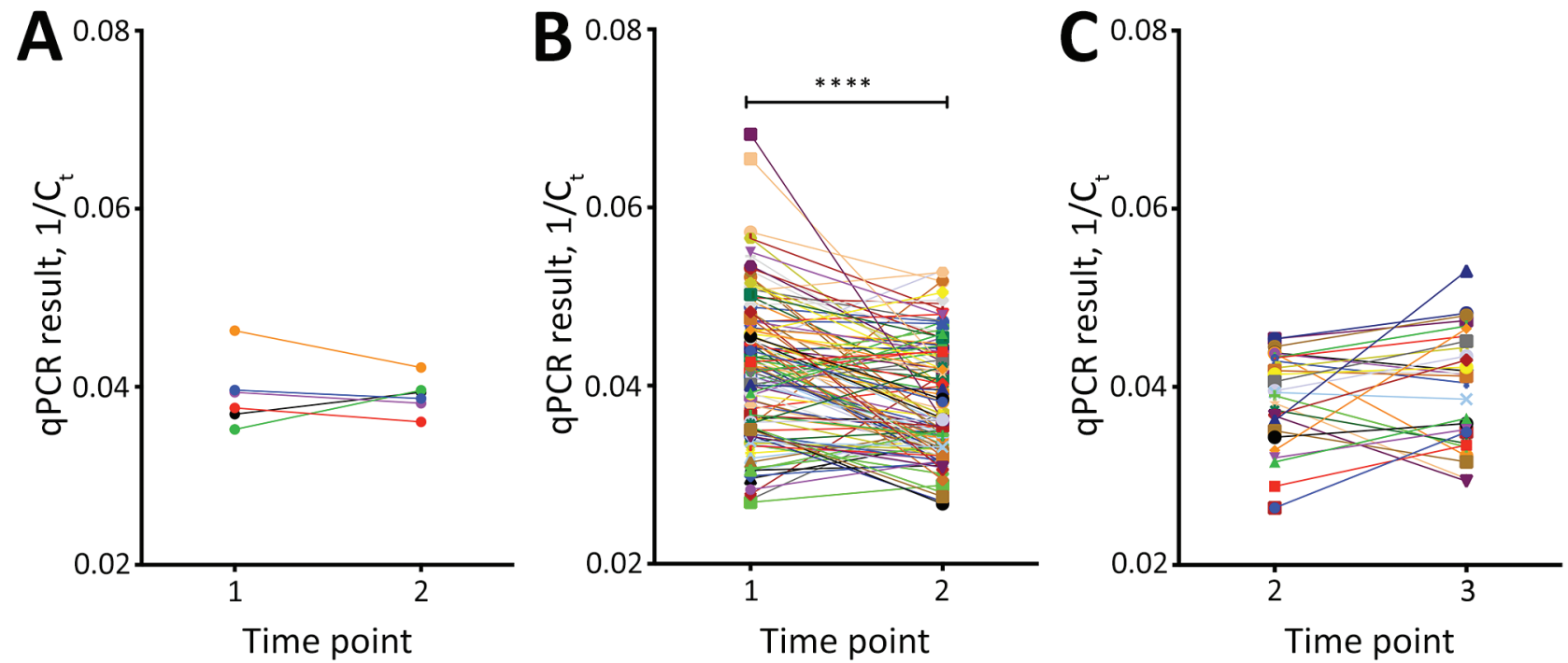

Figure 4. Change in relative quantities of Ancylostoma ceylanicum and Necator americanus hookworm genomic DNA in fecal samples from US-bound Myanmar refugees at 3 camps along the Myanmar-Thailand border after treatments with albendazole and ivermectin, Thailand, 2012-2015. Fecal samples were collected at 3 time points: time point 1 (T1, baseline), time point 2 (T2, after first treatment), and time point 3 (T3, after second treatment). Quantities were expressed as $1 / C_{t}$ and differences were assessed by paired $t$-test. A) $A$. ceylanicum genomic DNA relative quantities in those who were persistently infected after first treatment $(n=6)$. The difference between T1 and T2 was not significant. B) $N$. americanus genomic DNA relative quantities in those who were persistently infected after first treatment $(n=122)$. The relative genomic DNA quantity was significantly reduced in those who remained infected with $N$. americanus hookworm after first treatment. C) $N$. americanus genomic DNA relative quantities in those who were persistently infected after second treatment $(n=29)$. No significant change in $N$. americanus relative quantity was found in those persistently infected after the second treatment. The geometric mean time from T1 to T2 was 188.2 (range 48-1,013) d and from T2 to T3 was 46.2 (range 14-413) d. ${ }^{* * * *} \mathrm{p}<0.0001 . \mathrm{C}_{\mathrm{t}}$, cycle threshold; qPCR, quantitative PCR. 
DNA relative quantity at $\mathrm{T} 3$, and 26 refugees were positive for $N$. americanus hookworm at all 3 time points.

We detected a small number of newly acquired hookworm infections. Of the 1,548 participants with T1-T2 paired samples, we detected $15(\approx 1 \%)$ new $A$. ceylanicum infections and $36(2.3 \%)$ new $N$. americanus infections (Table 4). Of the 517 participants with T2-T3 paired samples, we detected $3(0.58 \%)$ new $A$. ceylanicum infections and $13(2.5 \%)$ new $N$. americanus infections.

\section{Risk Factors Associated with $\mathbf{N}$. americanus and $A$. ceylanicum Infection}

A generalized linear model was used to assess whether coincident helminth or protozoa infection, age, sex, or camp affected the risk for infection with $N$. americanus or $A$. ceylanicum hookworm at T1 (Table 5). The $y$-intercept for the model of $N$. americanus hookworm was 2.4 (95\% CI 2.14 2.69) and for A. ceylanicum hookworm 3.24 (95\% CI 2.77 3.74 ). The strongest predictors (ORs $>1.5$ and $p$ values $<0.0001$ ) of $N$. americanus infection were adult age (OR 3.83), T. trichiura infection (OR 1.90), and A. lumbricoides infection (OR 1.71). Female participants had a reduced odds of $N$. americanus infection (OR $0.68 ; \mathrm{p}<0.0001$ ). $N$. americanus infection was the only 1 of the 5 infections assessed that was associated with $A$. ceylanicum co-infection (OR 2.08; $\mathrm{p}=0.0018)$; female sex (OR 0.57; $<<0.0001)$ and residence in camp 1 (Mae La, OR 0.69; $\mathrm{p}=0.03$ ) were associated with reduced odds of $A$. ceylanicum infection.

We used a similar model that took age, sex, and camp into account to compare participants who cleared their infection with $N$. americanus hookworm after 1 treatment (n $=290$ ) with those who did not clear infection after 2 treatments $(n=26)$. Female sex, but not age or camp, was associated with clearance after a single treatment (OR 2.14, $95 \%$ CI $1.23-4.44 ; \mathrm{p}=0.0045$ ).

\section{$N$. americanus Benzimidazole Resistance and $\beta$-Tubulin SNP Changes}

Because 26 participants were positive for $N$. americanus hookworm at all 3 time points despite 2 courses of albendazole treatment, benzimidazole drug resistance was a concern. In total, 19 of 26 persistently infected participants had T1 fecal samples available for DNA reextraction and SNP200 testing; 3 samples showed no amplification, and 16 were wild type. We then performed DNA reextraction and SNP200 and SNP167 testing with the T3 fecal samples available $(n=24)$. Only 11 of 24 samples had sufficient quantities of $N$. americanus DNA $(>150 \mathrm{pg} /$ $\mu \mathrm{L})$ to be amplified. All samples were positive for wild -type SNP200 and SNP167. None were homozygous or heterozygous for mutant alleles. Thus, no alterations in the $\beta$-tubulin gene could be detected at codons 167 or 200 to account for drug resistance.
An additional 213 samples (from multiple time points) had sufficient $N$. americanus DNA to test SNP200 variation further; 173 of 213 samples were evaluable by allelic discrimination qPCR. All were homozygous wild type for SNP200.

\section{Blood Cell Concentration Differences Between $\boldsymbol{N}$. americanus and $A$. ceylanicum Infections}

We further evaluated refugees with either $N$. americanus $(\mathrm{n}=143)$ or A. ceylanicum $(\mathrm{n}=24)$ hookworm monoinfections; participants co-infected with both hookworms or other soil-transmitted helminths (i.e., S. stercoralis, A. lumbricoides, and T. trichiura roundworms) were excluded. Peripheral blood eosinophil concentrations were significantly higher $(\mathrm{p}<0.001)$ in those with $A$. ceylanicum monoinfections (geometric mean $8.49 \times 10^{8}$ cells $/ \mathrm{L}, 95 \%$ CI 5.98-12.04 $\times 10^{8}$ cells $/ \mathrm{L}$ ) than those with $N$. americanus monoinfections (geometric mean $3.44 \times 10^{8}$ cells $/ \mathrm{L}, 95 \%$ CI 2.92-4.05 $\times 10^{8}$ cells/L) (Figure 5, panel A). The hemoglobin levels did not differ between those with only $A$. ceylanicum and those with only $N$. americanus infections (Figure 5, panel B).

\section{Discussion}

A. ceylanicum hookworm is increasingly being recognized as a pathogen in humans, particularly in Southeast Asia (32). A. ceylanicum is the only hookworm species known to achieve patency in both humans and other animals (e.g., dogs and cats) (33). In this group of US-bound refugees from Myanmar, $N$. americanus infection was the most prevalent hookworm infection at all 3 time points tested (25.4\% at baseline); A. ceylanicum was the only other hookworm species found, with a baseline prevalence of $5.4 \%$. Because A. ceylanicum infection has been described in both Thailand $(7,10)$ and Myanmar $(11,34)$, whether these persons were infected in their home country or in the camps in Thailand is unknown. Camp allocation seemed to have an effect on infection status for both hookworms; camps 2 and 3 imparted a higher risk for $A$. ceylanicum hookworm acquisition, and camp 1 had a higher rate of $N$. americanus infection. Evidence of newly acquired infections for both hookworms at T2 and T3 (Table 4) indicates that active transmission of both species was ongoing at these 3 camps. However, whether the majority of hookworm infections at baseline were acquired prior to entry or while residing in the camps is unknown. Differential infection rates at the 3 camps might reflect environmental and hygiene conditions in the camps, the historical exposure of the persons at these camps, or other factors. Of note, a risk factor for infection with either A. ceylanicum or $N$. americanus hookworm was infection with the other hookworm (Table 5). This finding contrasts with previous surveys showing that $A$. ceylanicum and $N$. americanus co-infections are rare $(26,32)$. Although 
Table 4. New hookworm infections acquired by US-bound Myanmar refugees in camps along Myanmar-Thailand border, by time point, Thailand, 2012-2015*

\begin{tabular}{lcc}
\hline Species & T2, $\mathrm{n}=1,548$, & $\mathrm{T} 3, \mathrm{n}=517$, \\
no. $(\%)$ & no. $(\%)$ \\
\hline Ancylostoma ceylanicum & $15(0.97)$ & $3(0.58)$ \\
Necator americanus & $36(2.3)$ & $13(2.5)$ \\
\hline${ }^{*}$ T2, time point 2; T3, time point 3. & & \\
\hline
\end{tabular}

A. ceylanicum infection by the fecal-oral route is thought to be possible (33), walking barefoot has been shown to be a major risk factor for $A$. ceylanicum and $N$. americanus hookworm infections (35). Thus, the high propensity for co-infection in the population we evaluated suggests a similar mode of transmission, namely transdermal penetration, for both hookworms.

Despite possible co-transmission, those infected with A. ceylanicum hookworm had slightly different risk factors than those infected with $N$. americanus hookworm. $A$. ceylanicum hookworm prevalence peaked in the third decade of life, compared with $N$. americanus prevalence, which peaked in the sixth and seventh decades. However, $N$. americanus prevalence remained high for many decades of life (20-69 years of age).

T. trichiura roundworm, Entamoeba histolytica ame$\mathrm{ba}$, and A. lumbricoides roundworm infection as risk factors for $N$. americanus hookworm co-infection reflects the high prevalence of parasitic infections in this population (21), in a setting with inadequate sanitary infrastructure despite improvement efforts (36). Why infections with these pathogens but not $S$. stercoralis roundworm (acquired similarly to hookworm) or Giardia duodenalis protozoa (acquired similarly to $E$. histolytica ameba) put refugees at risk for $N$. americanus infection deserves further study.

Female participants were less likely to acquire both $N$. americanus and A. ceylanicum hookworms, a finding that might reflect differential exposures, differences in immunity $(37,38)$, differences in albendazole metabolism (39), or a combination of these factors. Although eosinophilia has been previously reported in experimental human infections with A. ceylanicum hookworm (33) and in A. ceylanicum case reports $(34,40)$, a more striking eosinophilia was seen among those with $A$. ceylanicum infections than those with $N$. americanus infections. This observation supports the idea that, unlike the hookworms that only infect humans, the zoonotic A ceylanicum parasite might be less able to downregulate the host's IgE-mediated response to infection (41). No difference in hemoglobin levels was found between those with either hookworm species. Anemia is typically seen in A. duodenale infection and is less commonly associated with $N$. americanus infection (3); for $A$. ceylanicum infection, data on anemia are scant.

The treatment for $N$. americanus infection was only modestly effective (cure rate $42 \%-69 \%$ ). It has been suggested that deworming might have enabled the zoonotic $A$. ceylanicum hookworm to fill a niche left by a decrease in anthropophilic hookworms (32). That A. ceylanicum infection was largely cured after single courses of treatment with albendazole (cure rate $92.8 \%-100 \%$ ) is reassuring, although follow-up time periods in this evaluation were more varied than in most controlled studies specifically assessing response to treatment. Still, reinfection and newly acquired infection rates $(0.58 \%-0.97 \%$ for $A$. ceylanicum and $2.3 \%-2.5 \%$ for $N$. americanus) were modest after deworming, compared with previous projections suggesting reinfection rates as high as 30\% (14) after 3 months. With the average time between T1 and T2 exceeding 6 months (and the wide time range of 1-33 months), this population experienced a lower reinfection rate than has been suggested for endemic areas.

Persons who were infected with $N$. americanus hookworm at all 3 time points represent a group that might have never cleared the infection or might have cleared infection but were subsequently reinfected. However, the time between sample collections was not statistically different between those who were persistently positive and those who cleared infection after a single treatment (E.M. O'Connell,

Table 5. Characteristics of US-bound Myanmar refugees in camps along Myanmar-Thailand border associated with increased risk for infection with Ancylostoma ceylanicum or Necator americanus hookworm, Thailand, 2012-2015*

\begin{tabular}{|c|c|c|c|c|}
\hline \multirow[b]{2}{*}{ Category } & \multicolumn{2}{|c|}{ N. americanus } & \multicolumn{2}{|c|}{ A. ceylanicum } \\
\hline & Odds ratio & $p$ value & Odds ratio & $p$ value \\
\hline Children compared with infants and toddlers & 8.17 & 0.0025 & NS & NS \\
\hline Adults compared with children & 3.83 & $<0.0001$ & NS & NS \\
\hline Ancylostoma ceylanicum infection & 2.08 & 0.0017 & NA & NA \\
\hline Trichuris trichiura infection & 1.90 & $<0.0001$ & NS & NS \\
\hline Entamoeba histolytica infection & 1.79 & 0.0173 & NS & NS \\
\hline Ascaris lumbricoides infection & 1.71 & $<0.0001$ & NS & NS \\
\hline Residence at camp 1, Mae La & 1.27 & 0.0039 & 0.69 & 0.0303 \\
\hline Female sex & 0.68 & $<0.0001$ & 0.57 & $<0.0001$ \\
\hline Necator americanus infection & NA & NA & 2.08 & 0.0018 \\
\hline
\end{tabular}


Figure 5. Eosinophil and hemoglobin concentrations in US-bound Myanmar refugees at 3 camps along the Myanmar-Thailand border who were monoinfected with Ancylostoma ceylanicum (n $=24$ ) or Necator americanus $(n=143)$ hookworm at baseline, Thailand, 20122015. Those co-infected with both hookworms or Strongyloides stercoralis, Ascaris lumbricoides, or Trichuris trichiura roundworms were excluded from analysis. Horizontal line indicates geometric mean. Significance was calculated by MannWhitney test. A) The geometric mean eosinophil cell concentration was significantly higher in those with $A$. ceylanicum monoinfection $\left(8.49 \times 10^{8}\right.$ cells/L, $95 \% \mathrm{Cl}$ $5.98-12.0 \times 10^{8}$ cells/L) than those with $N$. americanus monoinfection $\left(3.44 \times 10^{8}\right.$ cells $\left./ \mathrm{L}, 95 \% \mathrm{Cl} 2.92-4.05 \times 10^{8} \mathrm{cells} / \mathrm{L}\right)\left({ }^{* * *} \mathrm{p}<0.001\right)$. B) The geometric mean hemoglobin level of the 2 groups was not significantly different.

unpub. data). Reports of benzimidazole resistance are increasing in the literature on veterinary medicine; resistance in canine Ancylostoma caninum hookworm (15) and phylogenetically similar bovine intestinal nematodes (17) was associated with SNPs in the $\beta$-tubulin gene, particularly in codon 200 but also in codon 167 and, rarely, in codon 198. Likewise, other studies have shown low cure rates after benzimidazole administration in the setting of $N$. americanus infection, and research suggests that resistance is emerging (42). One group found that albendazole administration exerts selective pressure on T. trichiura codon 200 in Kenya and Haiti (20). The same group found that, in pooled $N$. americanus eggs from Haiti, the allele containing the resistant codon 200 had a mean allelic frequency of $36 \%$ (43). A frequency of $0 \%$ was found for the homozygous resistant genotype in hookworm eggs (species not identified) from Haiti and Panama (20), and although the authors reported a $2.3 \%$ frequency of the homozygous resistant genotype in hookworm eggs from Kenya, the frequency of the resistant genotype after treatment did not increase (20), raising questions about the significance of this allele. Our examination of codons 167 and 200 in samples persistently positive for $N$. americanus hookworm across all 3 time points, and of codon 200 in those with high levels of $N$. americanus DNA in fecal samples at any time point, revealed only homozygous wild type $\beta$-tubulin genes.

Several possibilities might explain why $\beta$-tubulin mutations were not found to account for persistent $N$. americanus infections. First, the lesser-known codon 198 or another codon within the $\beta$-tubulin gene might be responsible for resistance in the hookworms infecting these refugees. Second, whereas amplification rates for our qPCR assay were as high as or higher than those in most other reports, up to $54 \%$ of samples positive for $N$. americanus DNA at T3 did not amplify adequately to determine genotype. Therefore, mutations in SNPs at either codon 167 or 200 could have been missed. Last, $\beta$-tubulin might not be the only gene involved in drug resistance and responsible for low cure rates in N. americanus. In this population, $88.5 \%$ (23 of 26) of those persistently positive for $N$. americanus at all 3 time points were male. The peak concentration in serum and area under the serum concentration time curve for albendazole sulfoxide and albendazole sulfone (the main active metabolites of albendazole) have been found to be higher in female than in male volunteers (39). Also, male refugees were possibly more likely than female refugees to quickly reacquire infection due to differences in environmental exposures.

One lesson from this evaluation was that unbiased surveys are necessary before pursuing highly specific molecular techniques when profiling organisms that infect a population, particularly when considering emerging infectious diseases. Although the use of the highly repetitive genomic sequence for $A$. duodenale hookworm is superior in sensitivity and specificity than the internal transcribed spacer 2 region (44), targeting the more conserved genomic region ultimately enabled detection of an unexpected organism, $A$. ceylanicum hookworm, which otherwise would have been missed in this population. 
This project had several limitations. Control and nontreatment groups were absent. Participation was completely voluntary. The number of fecal sample collections for the third time point dropped off substantially because of logistical issues relating to collecting and shipping samples from the various US states after resettlement. Unlike other studies focused on A. ceylanicum hookworm, this evaluation did not include surveying the local cat and dog populations to establish potential reservoirs, which might be a worthwhile future research direction.

In summary, this cohort of US-bound refugees living in 3 camps in Thailand on the Myanmar-Thailand border was found to have a high prevalence of $N$. americanus hookworm with suboptimal cure rates after albendazole administration that do not seem to be attributable to mutations in the $\beta$-tubulin gene at codons 200 or 167 . In addition, A. ceylanicum hookworm was the only other hookworm species identified in this population. A. ceylanicum infection had a much higher cure rate after a single course of albendazole, and those with $A$. ceylanicum monoinfection had a similar hemoglobin level as those with $N$. americanus monoinfection. Future mapping efforts of soil-transmitted helminths should take into account the emergence of $A$. ceylanicum hookworm infection in humans to further understand its distribution across the world. The recognition of the increased importance of zoonotic A. ceylanicum hookworm over that of $A$. duodenale hookworm in some populations raises epidemiologic questions about transmission dynamics and the differential effect on local health of these 2 species.

\section{Acknowledgments}

We thank Diana Martin and Chaohua Li for help in processing samples. We thank Marla Petway for assistance in specimen management.

This project was funded in part through the Division of Intramural Research, National Institute of Allergy and Infectious Diseases, Bethesda, Maryland, USA, and the Centers for Disease Control and Prevention, Atlanta, Georgia, USA.

\section{About the Author}

Dr. O'Connell is an assistant clinical investigator in the Laboratory of Parasitic Diseases, National Institute of Allergy and Infectious Diseases, National Institutes of Health, Bethesda, Maryland, USA. Her primary research interests are in helminthic infections; she works on the development of biomarkers and deciphering the host-parasite interactions in neurocysticercosis.

\section{References}

1. Vos T, Allen C, Arora M, Barber RM, Bhutta ZA, Brown A, et al.; Global Burden of Disease 2015 Disease and Injury Incidence and Prevalence Collaborators. Global, regional, and national incidence, prevalence, and years lived with disability for 310 diseases and injuries, 1990-2015: a systematic analysis for the Global
Burden of Disease Study 2015. Lancet. 2016;388:1545-602. http://dx.doi.org/10.1016/S0140-6736(16)31678-6

2. Papier K, Williams GM, Luceres-Catubig R, Ahmed F, Olveda RM, McManus DP, et al. Childhood malnutrition and parasitic helminth interactions. Clin Infect Dis. 2014;59:234-43. http://dx.doi.org/ $10.1093 / \mathrm{cid} / \mathrm{ciu} 211$

3. Jonker FA, Calis JC, Phiri K, Brienen EA, Khoffi H, Brabin BJ, et al. Real-time PCR demonstrates Ancylostoma duodenale is a key factor in the etiology of severe anemia and iron deficiency in Malawian pre-school children. PLoS Negl Trop Dis. 2012;6:e1555. http://dx.doi.org/10.1371/journal.pntd.0001555

4. Hoagland KE, Schad GA. Necator americanus and Ancylostoma duodenale: life history parameters and epidemiological implications of two sympatric hookworms of humans. Exp Parasitol. 1978;44:36-49. http://dx.doi.org/10.1016/0014-4894(78)90078-4

5. Mahdy MA, Lim YA, Ngui R, Siti Fatimah MR, Choy SH, Yap NJ, et al. Prevalence and zoonotic potential of canine hookworms in Malaysia. Parasit Vectors. 2012;5:88. http://dx.doi.org/10.1186/1756-3305-5-88

6. Liu Y, Zheng G, Alsarakibi M, Zhang X, Hu W, Lu P, et al. Molecular identification of Ancylostoma caninum isolated from cats in southern China based on complete ITS sequence. BioMed Res Int. 2013;2013:868050. http://dx.doi.org/10.1155/2013/868050

7. Pumidonming W, Salman D, Gronsang D, Abdelbaset AE, Sangkaeo K, Kawazu SI, et al. Prevalence of gastrointestinal helminth parasites of zoonotic significance in dogs and cats in lower northern Thailand. J Vet Med Sci. 2016;78:1779-84. http://dx.doi.org/10.1292/jvms.16-0293

8. Hu W, Yu XG, Wu S, Tan LP, Song MR, Abdulahi AY, et al. Levels of Ancylostoma infections and phylogenetic analysis of cox 1 gene of A. ceylanicum in stray cat faecal samples from Guangzhou, China. J Helminthol. 2016;90:392-7. http://dx.doi.org/10.1017/ S0022149X15000413

9. Smout FA, Skerratt LF, Butler JRA, Johnson CN, Congdon BC, Thompson RCA. The hookworm Ancylostoma ceylanicum: an emerging public health risk in Australian tropical rainforests and indigenous communities. One Health. 2017;3:66-9. http://dx.doi.org/10.1016/j.onehlt.2017.04.002

10. Phosuk I, Intapan PM, Thanchomnang T, Sanpool O, Janwan P, Laummaunwai P, et al. Molecular detection of Ancylostoma duodenale, Ancylostoma ceylanicum, and Necator americanus in humans in northeastern and southern Thailand. Korean J Parasitol. 2013;51:747-9. http://dx.doi.org/10.3347/kjp.2013.51.6.747

11. Pa Pa Aung W, Htoon TT, Tin HH, Sanpool O, Jongthawin J, Sadaow L, et al. First molecular identifications of Necator americanus and Ancylostoma ceylanicum infecting rural communities in lower Myanmar. Am J Trop Med Hyg. 2017; 96:214-6. http://dx.doi.org/10.4269/ajtmh.16-0610

12. Bradbury RS, Hii SF, Harrington H, Speare R, Traub R. Ancylostoma ceylanicum hookworm in the Solomon Islands. Emerg Infect Dis. 2017;23:252-7. http://dx.doi.org/10.3201/eid2302.160822

13. Ngui R, Ching LS, Kai TT, Roslan MA, Lim YA. Molecular identification of human hookworm infections in economically disadvantaged communities in peninsular Malaysia. Am J Trop Med Hyg. 2012;86:837-42. http://dx.doi.org/10.4269/ajtmh.2012.11-0446

14. Jia TW, Melville S, Utzinger J, King CH, Zhou XN. Soiltransmitted helminth reinfection after drug treatment: a systematic review and meta-analysis. PLoS Negl Trop Dis. 2012;6:e1621. http://dx.doi.org/10.1371/journal.pntd.0001621

15. Furtado LF, Bello AC, dos Santos HA, Carvalho MR, Rabelo EM. First identification of the F200Y SNP in the $\beta$-tubulin gene linked to benzimidazole resistance in Ancylostoma caninum. Vet Parasitol. 2014;206:313-6. http://dx.doi.org/10.1016/j.vetpar.2014.10.021

16. Albonico M, Wright V, Bickle Q. Molecular analysis of the $\beta$-tubulin gene of human hookworms as a basis for possible benzimidazole resistance on Pemba Island. Mol Biochem Parasitol. 
2004;134:281-4. http://dx.doi.org/10.1016/j.molbiopara. 2003.12.008

17. Demeler J, Krüger N, Krücken J, von der Heyden VC, Ramünke S, Küttler U, et al. Phylogenetic characterization of $\beta$-tubulins and development of pyrosequencing assays for benzimidazole resistance in cattle nematodes. PLoS One. 2013;8:e70212. http://dx.doi.org/10.1371/journal.pone.0070212

18. Hansen TV, Thamsborg SM, Olsen A, Prichard RK, Nejsum P. Genetic variations in the beta-tubulin gene and the internal transcribed spacer 2 region of Trichuris species from man and baboons. Parasit Vectors. 2013;6:236. http://dx.doi.org/10.1186/ 1756-3305-6-236

19. Schwenkenbecher JM, Albonico M, Bickle Q, Kaplan RM. Characterization of beta-tubulin genes in hookworms and investigation of resistance-associated mutations using real-time PCR. Mol Biochem Parasitol. 2007;156:167-74. http://dx.doi.org/ 10.1016/j.molbiopara.2007.07.019

20. Diawara A, Halpenny CM, Churcher TS, Mwandawiro C, Kihara J, Kaplan RM, et al. Association between response to albendazole treatment and $\beta$-tubulin genotype frequencies in soil-transmitted helminths. PLoS Negl Trop Dis. 2013;7:e2247. http://dx.doi.org/10.1371/journal.pntd.0002247

21. Mitchell T, Lee D, Weinberg M, Phares C, James N, Amornpaisarnloet K, et al. Impact of enhanced health interventions for United States-bound refugees: evaluating best practices in migration health. Am J Trop Med Hyg. 2018;98:920-8. http://dx.doi.org/10.4269/ajtmh.17-0725

22. Easton AV, Oliveira RG, O'Connell EM, Kepha S, Mwandawiro CS, Njenga SM, et al. Multi-parallel qPCR provides increased sensitivity and diagnostic breadth for gastrointestinal parasites of humans: field-based inferences on the impact of mass deworming. Parasit Vectors. 2016;9:38. http://dx.doi.org/10.1186/s13071-016-1314-y

23. Basuni M, Muhi J, Othman N, Verweij JJ, Ahmad M, Miswan N, et al. A pentaplex real-time polymerase chain reaction assay for detection of four species of soil-transmitted helminths. Am J Trop Med Hyg. 2011;84:338-43. http://dx.doi.org/10.4269/ ajtmh.2011.10-0499

24. Pilotte N, Papaiakovou M, Grant JR, Bierwert LA, Llewellyn S, McCarthy JS, et al. Improved PCR-based detection of soil transmitted helminth infections using a next-generation sequencing approach to assay design. PLoS Negl Trop Dis. 2016;10:e004578. http://dx.doi.org/10.1371/journal.pntd.0004578

25. Papaiakovou M, Pilotte N, Grant JR, Traub RJ, Llewellyn S, McCarthy JS, et al. A novel, species-specific, real-time PCR assay for the detection of the emerging zoonotic parasite Ancylostoma ceylanicum in human stool. PLoS Negl Trop Dis. 2017;11:e0005734. http://dx.doi.org/10.1371/journal.pntd.0005734

26. George S, Kaliappan SP, Kattula D, Roy S, Geldhof P, Kang G, et al. Identification of Ancylostoma ceylanicum in children from a tribal community in Tamil Nadu, India using a semi-nested PCR-RFLP tool. Trans R Soc Trop Med Hyg. 2015;109:283-5. http://dx.doi.org/10.1093/trstmh/trv001

27. Lang AH, Drexel H, Geller-Rhomberg S, Stark N, Winder T, Geiger K, et al. Optimized allele-specific real-time PCR assays for the detection of common mutations in KRAS and BRAF. J Mol Diagn. 2011;13:23-8. http://dx.doi.org/10.1016/ j.jmoldx.2010.11.007

28. Makanga JO, Christianto A, Inazu T. Allele-specific real-time polymerase chain reaction as a tool for urate transporter 1 mutation detection. Methods Mol Biol. 2015;1275:117-25. http://dx.doi.org/ 10.1007/978-1-4939-2365-6_8

29. Tol J, Dijkstra JR, Vink-Börger ME, Nagtegaal ID, Punt CJ, Van Krieken JH, et al. High sensitivity of both sequencing and real-time PCR analysis of KRAS mutations in colorectal cancer tissue. J Cell Mol Med. 2010;14:2122-31. http://dx.doi.org/ 10.1111/j.1582-4934.2009.00788.x
30. Tulve NS, Suggs JC, McCurdy T, Cohen Hubal EA, Moya J. Frequency of mouthing behavior in young children. J Expo Anal Environ Epidemiol. 2002;12:259-64. http://dx.doi.org/10.1038/ sj.jea. 7500225

31. Mattioli MC, Davis J, Boehm AB. Hand-to-mouth contacts result in greater ingestion of feces than dietary water consumption in Tanzania: a quantitative fecal exposure assessment model. Environ Sci Technol. 2015;49:1912-20. http://dx.doi.org/10.1021/es505555f

32. Inpankaew T, Schär F, Dalsgaard A, Khieu V, Chimnoi W, Chhoun C, et al. High prevalence of Ancylostoma ceylanicum hookworm infections in humans, Cambodia, 2012. Emerg Infect Dis. 2014;20:976-82. http://dx.doi.org/10.3201/eid2006.131770

33. Wijers DJ, Smit AM. Early symptoms after experimental infection of man with Ancylostoma braziliense var. ceylanicum. Trop Geogr Med. 1966;18:48-52.

34. Brunet J, Lemoine JP, Lefebvre N, Denis J, Pfaff AW, Abou-Bacar A, et al. Bloody diarrhea associated with hookworm infection in traveler returning to France from Myanmar. Emerg Infect Dis. 2015;21:1878-9. http://dx.doi.org/10.3201/eid2110.150695

35. Ngui R, Lim YA, Traub R, Mahmud R, Mistam MS. Epidemiological and genetic data supporting the transmission of Ancylostoma ceylanicum among human and domestic animals. PLoS Negl Trop Dis. 2012;6:e1522. http://dx.doi.org/10.1371/journal.pntd.0001522

36. Phares CR, Date K, Travers P, Déglise C, Wongjindanon N, Ortega L, et al. Mass vaccination with a two-dose oral cholera vaccine in a long-standing refugee camp, Thailand. Vaccine. 2016;34:128-33. http://dx.doi.org/10.1016/j.vaccine.2015.10.112

37. Scott JT, Diakhaté M, Vereecken K, Fall A, Diop M, Ly A, et al. Human water contacts patterns in Schistosoma mansoni epidemic foci in northern Senegal change according to age, sex and place of residence, but are not related to intensity of infection. Trop Med Int Health. 2003;8:100-8. http://dx.doi.org/10.1046/j.1365-3156.2003.00993.x

38. Sow S, de Vlas SJ, Stelma F, Vereecken K, Gryseels B, Polman K. The contribution of water contact behavior to the high Schistosoma mansoni infection rates observed in the Senegal River basin. BMC Infect Dis. 2011;11:198. http://dx.doi.org/10.1186/1471-2334-11-198

39. Mirfazaelian A, Dadashzadeh S, Rouini M. Effect of gender in the disposition of albendazole metabolites in humans. Eur J Clin Pharmacol. 2002;58:403-8. http://dx.doi.org/10.1007/ s00228-002-0488-8

40. Hsu YC, Lin JT. Images in clinical medicine. Intestinal infestation with Ancylostoma ceylanicum. N Engl J Med. 2012;366:e20. http://dx.doi.org/10.1056/NEJMicm1101717

41. Traub RJ. Ancylostoma ceylanicum, a re-emerging but neglected parasitic zoonosis. Int J Parasitol. 2013;43:1009-15. http://dx.doi.org/10.1016/j.ijpara.2013.07.006

42. Humphries D, Simms BT, Davey D, Otchere J, Quagraine J, Terryah S, et al. Hookworm infection among school age children in Kintampo North municipality, Ghana: nutritional risk factors and response to albendazole treatment. Am J Trop Med Hyg. 2013;89:540-8. http://dx.doi.org/10.4269/ajtmh.12-0605

43. Diawara A, Schwenkenbecher JM, Kaplan RM, Prichard RK. Molecular and biological diagnostic tests for monitoring benzimidazole resistance in human soil-transmitted helminths. Am J Trop Med Hyg. 2013;88:1052-61. http://dx.doi.org/10.4269/ ajtmh.12-0484

44. Pilotte N, Papaiakovou M, Grant JR, Bierwert LA, Llewellyn S, McCarthy JS, et al. Improved PCR-based detection of soil transmitted helminth infections using a next-generation sequencing approach to assay design. PLoS Negl Trop Dis. 2016;10:e0004578. http://dx.doi.org/10.1371/journal.pntd.0004578

Address for correspondence: Elise M. O'Connell, National Institute of Allergy and Infectious Diseases, 4 Center Dr, B105, Bethesda, MD 20892-6612, USA; email: oconnellem@niaid.nih.gov 